\title{
APPROXIMATION OF CONTINUOUS FUNCTIONS BY TYPICAL MEANS OF THEIR FOURIER SERIES
}

\author{
S. ALJANČIĆ
}

1. Notations and results. (i) Let $C$ be the class of continuous and periodic functions $f$ of period $2 \pi$ such that $\int_{0}^{2 \pi} f(x) d x=0$.

The Lipschitz and Zygmund class $M^{1} \Lambda_{\alpha}$ and $M^{2} \Lambda_{\alpha}$ or simply ${ }^{1} \Lambda_{\alpha}$ and ${ }^{2} \Lambda_{\alpha} \quad(0<\alpha \leqq 1)$ are defined by $|f(x+h)-f(x)| \leqq M|h|^{\alpha}$ and $|f(x+h)+f(x-h)-2 f(x)| \leqq M|h|^{\alpha}$ respectively. Though ${ }^{1} \Lambda_{\alpha}={ }^{2} \Lambda_{\alpha}$ if $0<\alpha<1$, it is sometimes convenient to preserve for $0<\alpha<1$ both notations, even the neutral one $\Lambda_{\alpha}$.

By $M W^{r}(\beta)$ or simply by $W^{r}(\beta), r>0, \beta$ real, we denote the class of functions $f \in C$ whose representation is

$$
f(x)=\frac{1}{\pi} \int_{0}^{2 \pi} \phi(t) \psi_{r}(x-t ; \beta) d t
$$

where

$$
\psi_{r}(x ; \beta)=\sum_{\nu=1}^{\infty} \frac{\cos (\nu x-\beta \pi / 2)}{\nu^{r}}
$$

and

$$
\int_{0}^{2 \pi} \phi(x) d x=0, \quad \sup _{0 \leqq x \leqq 2 \pi} \operatorname{ess}|\phi(x)| \leqq M
$$

(Stečkin [7]). Two particular cases of the class $W^{r}(\beta)$ are of special interest: $W^{r}(r)=W^{r}$ and $W^{r}(0)$ (Nikolsky [6], B. Sz.-Nagy [5]). If, for simplicity, we suppose $0<r<1, W^{r}$ is the class of functions whose fractional integral $f_{1-r}$ is absolutely continuous and whose fractional derivative $f^{(r)}=\phi$ almost everywhere. We notice that the class $W^{r}$ stands in the same relation to the class $\Lambda_{r}$ as the class ${ }^{1} \Lambda_{1}$ to ${ }^{2} \Lambda_{1}$. Indeed, by definition, $f \in W^{r}$ is equivalent to $f_{1-r} \in{ }^{1} \Lambda_{1}$ and by [10a, Chapter XII, Theorems 8.13 and 8.14], $f \in \Lambda_{r}$ is equivalent to $f_{1-r} \in{ }^{2} \Lambda_{1}$. Hence, there is the strict inclusion $W^{r} \subset \Lambda_{r}(0<r<1)$, which together with $W^{1}={ }^{1} \Lambda_{1}$ can be written in the form $W^{r} \subset{ }^{2} \Lambda_{r}(0<r \leqq 1){ }^{1}$ Similarly $W^{r}(0)$ can be defined by

Received by the editors October 24, 1960.

1 In many statements $W^{r}(0<r<1)$ appears as the natural continuation of the class ${ }^{1} \Lambda_{1}$. For instance: if $f \in W^{r}$ or $f \in{ }^{2} \Lambda_{r}(0<r \leqq 1)$ the Fourier coefficients of $f$ are $o\left(n^{-r}\right)$ or $O\left(n^{-r}\right)$ respectively. 


$$
\left(f \cos \frac{r \pi}{2}+\tilde{f} \sin \frac{r \pi}{2}\right)_{1-r} \in \in^{1} \Lambda_{1} \quad(0<r \leqq 1)
$$

where $\tilde{f}$ is the conjugate function to $f$, and there is the strict inclusion $W^{r}(0) \subset^{2} \Lambda_{r}, 0<r \leqq 1$. The classes $W^{r}$ and $W^{r}(0)$ are essentially first difference classes and so they are not reflexive in the sense of conjugacy. The conjugate classes $\bar{W}^{r}$ and $\bar{W}^{r}(0)$ are defined by $\tilde{f} \in W^{r}$ and $\tilde{f} \in W^{r}(0)$ respectively.

(ii) Let $\mathfrak{I}_{n}(f)=\mathfrak{T}_{n}(f ; x)$ be a method of approximation which to each $f \in C$ coordinates a trigonometrical polynomial of order $\leqq n$. Let $\mathfrak{M} \subset C$ and let

$$
\Delta_{n}(f)=\Delta_{n}(f ; \mathfrak{T})=\max _{0 \leqq x \leqq 2 \pi}\left|f(x)-\mathfrak{T}_{n}(f ; x)\right| .
$$

There are three different questions to be answered: (1) given a class $\mathfrak{M}$, to find an estimate of $\Delta_{n}(f)$ if $f \in \mathfrak{M}$ (a direct theorem); (2) given a positive function $\phi(n)$, with $\phi(n) \downarrow 0$ when $n \rightarrow \infty$, to determine the class $\mathfrak{M}$ such that $\Delta_{n}(f)=O[\phi(n)]$ if $f \in \mathfrak{M}$ (an inverse theorem); (3) to fix a class $\mathfrak{M}$ and a function $\phi(n)$ such that for the pair $(\mathfrak{M}, \phi)$ both the direct and the inverse theorem hold simultaneously (a theorem of equivalence).

There are such methods of approximation (Hille [4]) which, with strengthening the class $\mathfrak{M}$, give better approximation but only to a certain point. We say, with Favard [3], that a method of approximation $\mathfrak{I}_{n}(f)$ is saturated if there exist a class $\mathfrak{M}_{\mathfrak{T}}$ and a function $\phi_{\mathfrak{I}}(n)$ such that (1) for the pair $\left(\mathfrak{M}_{\mathfrak{T}}, \phi_{\mathfrak{T}}\right)$ holds an equivalence theorem and (2) if $\Delta_{n}(f ; \mathfrak{T})=O\left[\phi_{\mathfrak{T}}(n)\right]$ implies $f(x) \equiv 0 . \phi_{\mathfrak{T}}(n)$ is the order of best approximation of the method $\mathfrak{T}_{n}(f)$ and $\mathfrak{M T}$ is its class of saturation. Such an equivalence theorem is called the theorem of saturation for the method $\mathfrak{T}_{n}(f)$.

Let $T_{n}^{*}(f)$ be the trigonometrical polynomial of best approximation of order $\leqq n$ of the function $f$. $T_{n}^{*}(f)$ defines a method of approximation and in the usual notation $\Delta_{n}\left(f ; T^{*}\right)=E_{n}(f)$. If $k=0,1,2, \cdots$ and $0<\alpha \leqq 1$, then

$$
f^{(k)} \in{ }^{2} \Lambda_{\alpha} \Leftrightarrow E_{n}(f)=O\left(n^{-k-\alpha}\right) .
$$

For $0<\alpha<1$ this is the classical direct and inverse theorem of Jackson and Bernstein respectively. The case $\alpha=1$ is due to Zygmund [10c]. From his result followed the adequacy of the second difference class in equivalence theorems for $E_{n}(f)$.

The aim of this note is to point out that the Zygmund class plays a similar role in other methods of approximation. In order to see this, the familiar Fejér's sum $\sigma_{n}(f)$ is an inadequate method because it 
saturates with the best approximation of order just $1 / n$. Indeed, from Bernstein's, Alexits' [1] and Zamansky's [9a] result

$$
\left.\begin{array}{l}
f \in \Lambda_{\alpha}, 0<\alpha<1 \\
\tilde{f} \in{ }^{1} \Lambda_{1}
\end{array}\right\} \Leftrightarrow f-\sigma_{n}(f)=O\left(n^{-\alpha}\right)
$$

nothing can be said about the right interpretation of the class $\Lambda_{\alpha}$. Yet this result shows that there exists an essential difference between equivalence theorems which precede the saturation of the method and the one when the method just saturates.

An illustrative method of approximation from this point of view is the method of typical means

$$
R_{n}^{\lambda}(f)=\sum_{\nu=1}^{n-1}\left(1-\frac{\nu^{\lambda}}{n^{\lambda}}\right)\left(a_{\nu} \cos \nu x+b_{\nu} \sin \nu x\right)
$$

where $a_{v}$ and $b_{v}$ are Fourier coefficients of $f$. This method saturates and its best approximation is of order $n^{-\lambda}$.

Theorem. Let $k=0,1, \cdots$ and $0<\alpha \leqq 1$. Then

$1^{\circ} f-R_{n}^{\lambda}(f)=o\left(n^{-\lambda}\right) \Rightarrow f \equiv 0$;

$2^{\circ} f \in W^{\lambda}(0) \Leftrightarrow f-R_{n}^{\lambda}(f)=O\left(n^{-\lambda}\right)$;

$3^{\circ} f^{(k)} \in{ }^{2} \Lambda_{\alpha}, k+\alpha<\lambda \Leftrightarrow f-R_{n}^{\lambda}(f)=O\left(n^{-k-\alpha}\right)$.

Part $2^{\circ}$ is the saturation theorem for $R_{n}^{\lambda}(f)$.

REMARKs 1. A similar result holds for approximation of $\tilde{f}$ by $R_{n}^{\lambda}(\tilde{f})$. On account of the reflexivity of the class ${ }^{2} \Lambda_{\alpha}$, one has only to replace the class $W^{\lambda}(0)$ by its conjugate class $W^{\lambda}(0)$ in part $2^{\circ}$ of the theorem.

2. If the Fourier series of $f$ is of power series type, that is if $f \sim c_{1} e^{i x}$ $+c_{2} e^{2 i x}+\cdots$ and we put $R_{n}^{\lambda}(f)=\sum\left(1-\nu^{\lambda} / n^{\lambda}\right) c_{\nu} e^{\nu i x}$, then, on account of $\tilde{f}=-i f$, the theorem still holds; only the class $W^{\lambda}(0)$ in part $2^{\circ}$ is reduced to $W^{\lambda}$.

Some parts and some particular cases of the theorem are not new. Zygmund [10b] considered the case $\lambda=1,2, \cdots$ and his result is that for $k \leqq \lambda-1$

$$
f^{(k)} \in{ }^{1} \Lambda_{\alpha}, 0<\alpha \leqq 1 \Rightarrow f-R_{n}^{\lambda}(f)=O\left(n^{-k-\alpha}\right),
$$

except if $\lambda$ is odd and $k=\lambda-1, \alpha=1$ when in the $O$-term a $\log n$ is to be introduced. He remarked also that a similar result holds for the approximation of $\tilde{f}$ by $R_{n}^{\lambda}(\tilde{f})$, its critical case being $\lambda$ even and $\lambda=k-1, \alpha=1$. B. Sz.-Nagy [5] considered $\lambda>0$ (not necessarily integral) and obtained the direct theorem in part $2^{\circ}$. From his general results followed also various other direct theorems but only 
for classes of functions of first difference type. The saturation theorem for $\lambda=1,2, \cdots$ was proved by Zamansky [9b]. For $\lambda>0$ it was proved almost simultaneously and in different ways by Sunouchi and Watari [8] and by the author $[2 \mathrm{a}, \mathrm{b}]$ (the latter considered even typical means of the form $\sum\left(1-\lambda_{v} / \lambda_{n}\right) u_{v}$ where $\lambda_{n} \uparrow \infty$ and $\Delta^{2} \lambda_{n} \leqq 0$ or $\Delta^{2} \lambda_{n} \geqq 0$ with $\lambda_{2 n}=O\left(\lambda_{n}\right)$.

2. Proof of the theorem. It remains only to prove $3^{\circ}$ of the theorem. The inverse part in $3^{\circ}$ is a consequence of (1) because $f-R_{n}^{\lambda}(f)$ $=O\left(n^{-k-\alpha}\right)$ implies $E_{n}(f)=O\left(n^{-k-\alpha}\right)$. To prove the direct part in $3^{\circ}$, we prove first

(2) $f^{(k)} \in M^{1} \Lambda_{\alpha}\left(\begin{array}{c}k=0,1, \cdots ; 0 \leqq \alpha \leqq 1 \\ \lambda>k+\alpha\end{array}\right)$

$$
\Rightarrow\left|f-R_{n}^{\lambda}(f)\right| \leqq A_{\lambda, k, \alpha} M n^{-k-\alpha} .
$$

The passage to the class ${ }^{2} \Lambda_{\alpha}$ then follows by introduction of the moving average function, this device being slightly modified on account of the saturation of the method $R_{n}^{\lambda}(f)$, and using (2) and the somewhat more precise form of the direct part in $2^{\circ}$, namely

$$
f \in M W^{\lambda}(0) \Rightarrow\left|f-R_{n}^{\lambda}(f)\right| \leqq B_{\lambda} M n^{-\lambda} .
$$

(i) Obviously

$$
R_{n}^{\lambda}(f)-f=\frac{1}{\pi} \int_{0}^{\pi}[f(x+t)+f(x-t)-2 f(x)] K_{n}^{\lambda, 0}(t) d t,
$$

where

$$
K_{n}^{\lambda, 0}(t)=\frac{1}{2}+\sum_{\nu=1}^{n-1}\left(1-\frac{\nu^{\lambda}}{n^{\lambda}}\right) \cos \nu t
$$

Let

$$
\begin{aligned}
K_{n}^{\lambda, 1}(t) & =-\int_{t}^{\pi} K_{n}^{\lambda, 0}(u) d u=-\frac{\pi-t}{2}+\sum_{\nu=1}^{n-1}\left(1-\frac{\nu^{\lambda}}{n^{\lambda}}\right) \frac{\sin \nu t}{\nu} \\
& =-\sum_{\nu=n}^{\infty} \frac{\cos (\nu t-\pi / 2)}{\nu}-\frac{1}{n^{\lambda}} \sum_{\nu=1}^{n-1} \nu^{\lambda-1} \cos (\nu t-\pi / 2),
\end{aligned}
$$

the last equality being valid only for $0<t \leqq \pi$, and let $(k=2,3, \cdots)$

$$
K_{n}^{\lambda, k}(t)=-\sum_{\nu=n}^{\infty} \frac{\cos (\nu t-k \pi / 2)}{\nu^{k}}-\frac{1}{n^{\lambda}} \sum_{\nu=1}^{n-1} \nu^{\lambda-k} \cos (\nu t-k \pi / 2)
$$


be the successive primitives of $K_{n}^{\lambda, 1}(t)$. On account of

$$
\begin{aligned}
& K_{n}^{\lambda, 1}(0)=-\pi / 2, \quad K_{n}^{\lambda, 1}(\pi)=0 ; \\
& K_{n}^{\lambda, k}(0)=K_{n}^{\lambda, k}(\pi)=0 \quad \text { for } k=3,5,7, \cdots,
\end{aligned}
$$

successive partial integration gives

$$
\begin{aligned}
R_{n}^{\lambda}(f)-f & =-\frac{1}{\pi} \int_{0}^{\pi}\left[f^{\prime}(x+t)-f^{\prime}(x-t)\right] K_{n}^{\lambda, 1}(t) d t \\
& =\frac{1}{\pi} \int_{0}^{\pi}\left[f^{\prime \prime}(x+t)+f^{\prime \prime}(x-t)\right] K_{n}^{\lambda, 2}(t) d t \\
& =-\frac{1}{\pi} \int_{0}^{\pi}\left[f^{\prime \prime \prime}(x+t)-f^{\prime \prime \prime}(x-t)\right] K_{n}^{\lambda, 8}(t) d t
\end{aligned}
$$

Finally, using (6) again, one obtains

$$
\text { (7) } R_{n}^{\lambda}(f)-f=\left\{\begin{array}{r}
\frac{1}{\pi} \int_{0}^{\pi}\left[f^{(k)}(x+t)+f^{(k)}(x-t)-2 f^{(k)}(x)\right] K_{n}^{\lambda, k}(t) d t, \\
k=0,2,4, \cdots, \\
\frac{1}{\pi} \int_{0}^{\pi}\left[f^{(k)}(x-t)-f^{(k)}(x+t)\right] K_{n}^{\lambda, k}(t) d t, \\
k=1,3,5, \cdots .
\end{array}\right.
$$

For our purpose we need the following estimate

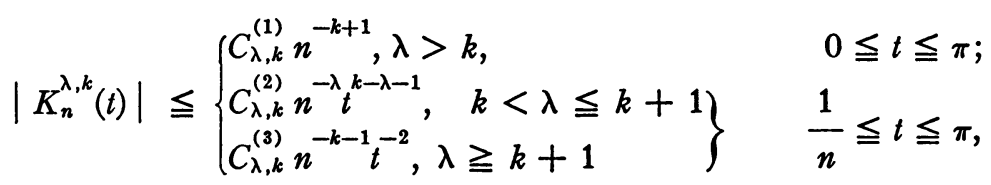

the proof of which is postponed to (iii).

Let first $0 \leqq \alpha<1$. We divide the integral in (7) in two: $\int_{0}^{1 / n}$ and $\int_{1 / n}^{\pi}$. $\left(8_{1}\right)$ gives $\pi\left|\int_{0}^{1 / n}\right| \leqq M(\alpha+1)^{-1} C_{\lambda, k}^{(1)} n^{-k-\alpha}$ and from $\left(8_{2}\right)$ and $\left(8_{3}\right)$ follows an estimate of the same type for $\pi\left|\int_{1 / n}^{\pi}\right|$, except that the constant $(a+1)^{-1} C_{\lambda, k}^{(1)}$ is to be replaced by $(\lambda-\alpha-k)^{-1} C_{\lambda, k}^{(2)}$ or $(1-\alpha)^{-1} C_{\lambda, k}^{(3)}$ according as $\lambda \leqq k+1$ or $\lambda \geqq k+1$. Hence, (2) is valid for $0 \leqq \alpha<1$.

Next, let $\alpha=1$. Then $f^{(k+1)}(x)$ exists and is bounded almost everywhere, so that this case is reduced to the preceding one with $\alpha=0$ and $k+1$ instead of $k$, which completes the proof of (2).

(ii) We now pass in (2) from the class ${ }^{1} \Lambda_{\alpha}$ to the class ${ }^{2} \Lambda_{\alpha}$. Only the case $\alpha=1$ requires a proof. For simplicity we consider only $k=0$, that is 


$$
f \in M^{2} \Lambda_{1} \Rightarrow f-R_{n}^{\lambda}(f)=O\left(n^{-1}\right),
$$

the proof for $k=1,2, \cdots$ being principally the same.

Let

$$
f_{\delta}(x)=\frac{1}{2 \delta} \int_{-\delta}^{\delta} f(x+t) d t=\frac{F(x+\delta)-F(x-\delta)}{2 \delta},
$$

where $F$ is a primitive of $f$, and let

$$
f_{\delta \delta}(x)=\frac{1}{4 \delta^{2}} \int_{-\delta}^{\delta} f_{\delta}(x+t) d t .
$$

If we put $f(x)=f_{\delta \delta}(x)+g(x)$, then

$$
|g(x)| \leqq M \delta / 2
$$

(see [10a, Chapter III, proof of Theorem 13.14]).

First, let $1<\lambda<2$. Using the known result [10a, Chapter XII, Theorem 8.14 (ii)]

$$
f \in M^{2} \Lambda_{1} \Rightarrow f^{(\gamma)} \in M_{\gamma}{ }^{1} \Lambda_{1-\gamma}, \quad 0<\gamma<1,
$$

and remembering that $f \in M^{2} \Lambda_{1} \Rightarrow f_{\delta} \in M^{2} \Lambda_{1}$ (with the same constant $M$ on both sides), we obtain

$$
\left|f_{\delta \delta}^{(\lambda)}(x)\right|=\left|\frac{f_{\delta}^{(\lambda-1)}(x+\delta)-f_{\delta}^{(\lambda-1)}(x-\delta)}{2 \delta}\right| \leqq \frac{M_{\lambda-1} \delta^{2-\lambda}}{2 \delta}=\frac{M_{\lambda-1}}{2} \delta^{1-\lambda} .
$$

The class ${ }^{2} \Lambda_{1}$ being reflexive, a similar estimate holds for $\tilde{f}_{\delta \delta}^{(\lambda)}(x)$, so that

$$
f_{\delta 8} \in D_{\lambda} \delta^{1-\lambda} W^{\lambda}(0)
$$

where $D_{\lambda}$ is independent on $\delta$.

Next, let $\lambda \geqq 2$. Then

$$
\begin{aligned}
\left|f_{\delta \delta}^{\prime \prime}(x)\right| & =\left|\frac{f_{\delta}^{\prime}(x+\delta)-f_{\delta}^{\prime}(x-\delta)}{2 \delta}\right| \\
& =\left|\frac{f(x+2 \delta)+f(x-2 \delta)-2 f(x)}{4 \delta^{2}}\right| \leqq \frac{M}{2 \delta},
\end{aligned}
$$

so that (10) holds for $\lambda=2$ too.

Now

$$
\left|f-R_{n}^{\lambda}(f)\right| \leqq\left|f_{\delta \delta}-R_{n}^{\lambda}\left(f_{\delta \delta}\right)\right|+\left|g-R_{n}^{\lambda}(g)\right|=\Delta_{1}+\Delta_{2} .
$$

Taking account of (9), (10) and (11), we apply (3), respectively (2), 
to $\Delta_{1}$ according as $1<\lambda \leqq 2$ or $\lambda>2$, and we apply (2) to $\Delta_{2}$ in both cases. This gives

$$
\left|f-R_{n}^{\lambda}(f)\right| \leqq B_{\lambda} D_{\lambda} \delta^{1-\lambda} n^{-\lambda}+A_{\lambda, 0,0} \frac{M}{2} \delta \quad \text { if } 1<\lambda \leqq 2,
$$

respectively

$$
\left|f-R_{n}^{\lambda}(f)\right| \leqq A_{\lambda, 2,0} \frac{M}{2 \delta} n^{-2}+A_{\lambda, 0,0} \frac{M}{2} \delta \quad \text { if } \lambda>2 .
$$

Putting here $\delta=\pi / 2 n$ in both cases follows $f-R_{n}^{\lambda}(f)=O\left(n^{-1}\right)$.

(iii) We sketch only the proof of (8), it being a matter of routine.

Let first $0 \leqq t \leqq \pi$. Then if $\lambda>k$ from $\left(5_{k}\right)(k=0,1,2, \cdots)$ follows

$$
\begin{aligned}
& \left|K_{n}^{\lambda, 0}(t)\right| \leqq \frac{1}{2}+\sum_{\nu=1}^{n-1} 1<n, \quad\left|K_{n}^{\lambda, 1}(t)\right| \leqq O(1)+n^{-\lambda} \sum_{\nu=1}^{n-1} \nu^{\lambda-1}=O(1), \\
& \left|K_{n}^{\lambda, k}(t)\right| \leqq \sum_{\nu=n}^{\infty} \nu^{-k}+n^{-\lambda} \sum_{\nu=1}^{n-1} \nu^{\lambda-k}=O\left(n^{-k+1}\right) \quad(k=2,3, \cdots) .
\end{aligned}
$$

Next, let $1 / n \leqq t \leqq \pi$ and $\lambda>k$ and put $p=[1 / t]$ and

$$
s_{n}=\frac{1}{2}+\sum_{\nu=1}^{n} \cos \nu t=\frac{\sin (n+1 / 2) t}{2 \sin t / 2} .
$$

Then

$$
\begin{aligned}
K_{n}^{\lambda, 0}(t) & =\frac{1}{n^{\lambda}} \sum_{\nu=0}^{n-1}\left[(\nu+1)^{\lambda}-\nu^{\lambda}\right] s_{\nu} \\
& =\operatorname{Im}\left\{\frac{e^{i t / 2}}{2 n^{\lambda} \sin t / 2} \sum_{\nu=0}^{n-1}\left[(\nu+1)^{\lambda}-\nu^{\lambda}\right] e^{\nu t i}\right\},
\end{aligned}
$$

so that

$$
\left|K_{n}^{\lambda, 0}(t)\right| \leqq \frac{1}{2 n^{\lambda} \sin t / 2}\left|\sum_{\nu=0}^{n-1}\left[(\nu+1)^{\lambda}-\nu^{\lambda}\right] e^{\nu t i}\right| .
$$

On the other hand, for $k=1,2, \cdots$, a partial summation gives

$$
\begin{aligned}
\left|K_{n}^{\lambda, k}(t)\right| \leqq & \left|\sum_{\nu=n}^{\infty} \nu^{-k} e^{\nu t i}+n^{-\lambda} \sum_{\nu=1}^{n-1} \nu^{\lambda-k} e^{\nu t i}\right| \\
\leqq & \frac{1}{2 \sin t / 2}\left\{\left|\sum_{\nu=n}^{\infty}\left[\nu^{-k}-(\nu+1)^{-k}\right] e^{\nu t i}\right|\right. \\
& \left.\quad+\left|n^{-\lambda} \sum_{\nu=0}^{n-1}\left[(\nu+1)^{\lambda-k}-\nu^{\lambda-k}\right] e^{\nu t i}\right|\right\} .
\end{aligned}
$$


We note that for $k=0$ the latter inequality formally reduces to (13).

The first term in (14) is $O\left(n^{-k-1} t^{-2}\right)$ (except for $k=0$ when it reduces to zero). The second term in (14) we divide in two: $\sum_{1}+\sum_{2}$ $=\left(2 n^{\lambda} \sin t / 2\right)^{-1}\left\{\left|\sum_{0}^{p-1}\right|+\left|\sum_{p}^{n-1}\right|\right\}$. Obviously, $\sum_{1}=O\left(n^{-\lambda} t^{-1} p^{\lambda-k}\right)$ $=O\left(n^{-\lambda} t^{k-\lambda-1}\right)$. The sequence $(\nu+1)^{\lambda-k}-\nu^{\lambda-k}$ being nonincreasing respectively nondecreasing as $\lambda \leqq k+1$ or $\lambda \geqq k+1$, we have $\sum_{2}$ $=O\left(n^{-\lambda} t^{-2}\right)\left[(p+1)^{\lambda-k}-p^{\lambda-k}\right]=O\left(n^{-\lambda} t^{k-\lambda-1}\right)$ respectively $\sum_{2}$ $=O\left(n^{-\lambda} t^{-2}\right)\left[(n+1)^{\lambda-k}-n^{\lambda-k}\right]=O\left(n^{-k-1} t^{-2}\right)$. Hence, in both cases

$$
K_{n}^{\lambda, k}(t)=O\left(n^{-k-1} t^{-2}\right)+O\left(n^{-\lambda} t^{k-\lambda-1}\right) .
$$

But, on account of $n t \geqq 1$, from $n^{-k-1} t^{-2}=n^{-\lambda} t^{k-\lambda-1} \cdot(n t)^{\lambda-k-1}$ follows that $n^{-k-1} t^{-2} \leqq n^{-\lambda} t^{k-\lambda-1}$ if $\lambda \leqq k+1$ respectively $n^{-k-1} t^{-2} \geqq n^{-\lambda} t^{k-\lambda-1}$ if $\lambda \geqq k+1$, so that $K_{n}^{\lambda, k}(t)=O\left(n^{-\lambda} t^{k-\lambda-1}\right)$ or $=O\left(n^{-k-1} t^{-2}\right)$ according as $k<\lambda \leqq k+1$ or $\lambda \geqq k+1$, which completes the proof of (8).

\section{REFERENCES}

1. G. Alexits, Sur l'ordre de grandeur de l'approximation d'une fonction par les moyennes de sa série de Fourier, Mat. Fiz. Lapok vol. 48 (1941) pp. 410-422.

2. S. Aljančić, (a) Classe de saturation des procédés de sommation de Hölder et de Riesz, C. R. Acad. Sci. Paris vol. 246 (1958) pp. 2567-2569. (b) Classe de saturation du procédé des moyennes typiques de Riesz, Acad. Serbe Sci. Publ. Inst. Math. vol. 13 (1959) pp. 113-122.

3. J. Favard, Colloques d'analyse harmonique, Nancy, Centre National de la Recherche Scientifique, Paris, vol. 15 (1949) pp. 97-110.

4. E. Hille, On the analytical theory of semi-groups, Proc. Nat. Acad. Sci. U.S.A. vol. 28 (1942) pp. 421-424.

5. B. Sz.-Nagy, Sur une classe générale de procédés de sommation pour les séries de Fourier, Hungarica Acta Math. vol. 1, no. 3 (1948) pp. 14-52.

6. S. M. Nikolsky, Approximation of periodic functions by trigonometrical polynomials (in Russian), Trudy Mat. Inst. Steklov vol. 15 (1945) 76 pp.

7. S. B. Stečkin, On the best approximation of some classes of periodic functions by trigonometric polynomials (in Russian), Izv. Akad. Nauk SSSR. Ser. Mat. vol. 20 (1956) pp. 643-648.

8. G. Sunouchi and C. Watari, On the determination of the class of saturation in the theory of approximation of functions, Proc. Japan Acad. vol. 34 (1958) pp. 477-481.

9. M. Zamansky, (a) Classes de saturation de certains procédés d'approximation des séries de Fourier, Ann. Sci. École Norm. Sup. vol. 66 (1949) pp. 19-93. (b) Classes de saturation des procédés de sommation des séries de Fourier et applications aux séries trigonométriques, Ann. Sci. Ecole Norm. Sup. vol. 67 (1950) pp. 161-198.

10. A. Zygmund, (a) Trigonometric series, 2d ed., Cambridge University Press, 1959. (b) The approximation of functions by typical means of their Fourier series, Duke Math. J. vol. 12 (1945) pp. 695-704. (c) Smooth functions, Duke Math. J. vol. 12 (1945) pp. 47-76.

Belgrade, Yugoslavia 